\title{
Strategies of intention formation are reflected in continuous MEG activity
}

\author{
Anja Achtziger \\ University of Konstanz, Germany \\ Thorsten Fehr \\ University of Bremen, Germany, and Otto von Guericke University of Magdeburg, Germany \\ Gabriele Oettingen \\ University of Hamburg, Germany, and New York University, NY, USA \\ Peter M. Gollwitzer \\ University of Konstanz, Germany, and New York University, NY, USA \\ Brigitte Rockstroh \\ University of Konstanz, Germany
}

\begin{abstract}
Self-regulation of intention formation is pivotal for achieving behavior change. Fantasy realization theory (Oettingen, 2000) assumes that mentally contrasting a desired positive future with present negative reality turns high expectations of success into strong intentions to realize the desired future, while indulging in the positive future fails to do so. The present study tests the theory's process assumption that mental contrasting is a cognitively demanding, purposeful problem-solving strategy involving working and episodic memory, whereas indulging is a mindless daydreaming strategy involving the free flow of thought, by investigating the neural correlates of the two strategies via continuous magnetoencephalographic (MEG) activity. We observed greater activity during mental contrasting (but not indulging) compared to resting in prefrontal, frontal, parietal, and temporal areas, indicating that mental contrasting involves strong intention formation, working memory, and episodic memory. In addition, heightened activity of occipital areas was observed during mental contrasting compared to resting and indulging, suggesting that mental contrasting, more than indulging and resting, entails purposefully creating mental images. Taken together, these findings indicate that mental contrasting is indeed a purposeful problemsolving strategy based on past performance history, whereas indulging is a purposeless daydreaming strategy that is oblivious to past experiences.
\end{abstract}

Correspondence should be addressed to: Anja Achtziger, University of Konstanz, Universitätsstr. 10, D-78464 Konstanz, Germany. E-mail: anja.achtziger@uni-konstanz.de

This research was supported by the Interdisciplinary Center for Research on Intentions and Intentionality (University of Konstanz), the Volkswagen-Foundation, and a German Research Foundation grant to Gabriele Oettingen (OE 237/5-1). We also thank Christina Bardong for her help in creating the pretest materials; Anne Häberle, Inge Schweiger Gallo, and Ursula Lommen for assistance in data acquisition; and Herrmann Hinrichs, Michael Scherg, and Thomas Elbert for methodological support. 


\section{INTRODUCTION}

In the attempt to explain its classic findings (extending from intrapersonal issues such as the attribution of success and failure to interpersonal issues such as the effects of stereotype threat on performance), social psychology has witnessed many debates between researchers taking a cognitive perspective vs. researchers adhering to a motivational approach (Sorrentino \& Higgins, 1986; Higgins \& Sorrentino, 1990). It is only recently that social psychologists of the two sides have joined forces in developing a comprehensive psychology of action (Gollwitzer \& Bargh, 1996), as explaining when and how actions are initiated, sustained, disrupted, and resumed requires the collaboration of cognitive and motivational researchers. In this endeavour, the concept of goals (intentions) turned out to be particularly useful; goals (intentions) are considered to be directors and energizers of action. In the study of how goals guide action, it became apparent that two questions need to be answered first (Oettingen \& Gollwitzer, 2001): What contents do people specify in their goals, and what processes lead to firm goal commitments? Whereas most social psychologists have focused on the contents of goals that are specified (e.g., promotion vs. prevention goals (Higgins, 1997); performance vs. learning goals (Dweck, 2000)) and their differential consequences for goal striving and goal attainment, few have analyzed the types of strategies used in goal setting (e.g., Oettingen, Pak, \& Schnetter, 2001) and their differential consequences for the strength of goal commitment.

The present study will focus on different strategies of goal setting and investigate their neural substrates. While research on intentions (goals) has become a central theme in social psychology, neuroscience has also seen an increase of investigations on the neural processes associated with intentions (e.g., Blakemore \& Frith, 2003). However, there is still a lack of neuroscience studies that explicitly investigate assumptions of intention (i.e., goal) theories as suggested by social psychologists. Neuroscientists and social psychologists have started various collaborations to attenuate this problem (summary by Morsella, Bargh, \& Gollwitzer, in press), and the present research is part of this endeavour.

\section{Fantasy realization theory}

For successful goal attainment, people need to first form the intention to reach a desired future outcome (i.e., firmly commit to goals) that they then effortfully and persistently strive for. Therefore, committing to feasible goals is important for goal attainment. Fantasy realization theory (Oettingen, 2000) has distinguished between two strategies that people can use to commit to goals of realizing a desired positive future: mental contrasting versus indulging. The two strategies differ to the extent to which they succeed in translating high perceived feasibility (expectations) of attaining the desired positive future into strong goal commitments. The strategy of mentally contrasting, which juxtaposes the desired positive future with obstacles of present reality, makes people consider expectations when forming goal commitments; thus, when expectations of successfully changing the present reality (mastering the obstacles) towards the desired future are high, strong commitments to realizing the desired future emerge. On the contrary, indulging in the desired future (i.e., merely daydreaming about it) makes people fail to use their expectations of success when forming goal commitments; thus, indulging leads to goal commitment independent of subjective probabilities of success. Even when expectations of success are high, only moderate goal commitments are observed.

When people use the self-regulation strategy of mental contrasting, they first imagine a desired future (e.g., improving in academic or professional performance) and then reflect on the corresponding negative reality (e.g., having little time or being distracted). The conjoint elaboration of the positive future and the negative reality makes both the future and the reality simultaneously accessible, whereby negative reality is perceived as standing in the way of realizing the desired future. In line with Newell and Simon's (1972) theory of problem solving, the model of fantasy realization conceives of a person who wants to change the present reality towards an imagined future as a person who faces the problem of wanting something and needing to engage in specific actions she can perform to attain it (p. 72). Accordingly, the objective problem space (defined as the objective task demands posed by the environment) entails the mental representation of both the positive future to be reached and the negative reality to be improved. If the 
subjective problem space (defined as the internal subjective representation of the problem at hand) matches the objective problem space, the person will recognize that she needs to act on the status quo in order to reach the desired future. Therefore, the perceived feasibility (expectations) of turning the present reality into the desired future will be regarded when forming respective intentions (i.e., making goal commitments). However, if the subjective problem space entails only a part of the objective problem space (e.g., only the positive future), the person will fail to recognize that she needs to change the status quo in order to arrive at the desired future. As a consequence, expectations of success are not regarded and the level of goal commitment is determined by the $a$ priori commitment that the person holds with respect to attaining the desired future.

A series of experimental studies measuring goal commitment as the dependent variable in various ways (i.e., via self-report but also via actual goal striving and attainment) supports these hypotheses. In Oettingen et al. (2001) participants either mentally elaborated both the desired future and negative reality (mental contrasting condition) or only the desired future (indulging condition). In one experiment (Study 4), adolescent students had to mentally contrast the positive future of excelling in mathematics (participants imagined, for example, feelings of pride, increasing job prospects) with the respective negative reality (participants reflected on, for example, being distracted by peers, feeling lazy). Two weeks after the experiment, students with high success expectations of excelling in math showed a higher goal commitment after mental contrasting than after indulging; that is, they achieved better course grades and teachers rated them as exerting more effort than students in the indulging condition. The same pattern of results emerged, for instance, in school children starting to learn a foreign language (Oettingen, Hönig, \& Gollwitzer, 2000, Study 1) or trying to better relate to minority peers (Oettingen, Mayer, Thorpe, Janetzke, \& Lorenz, 2005, Study 2), but also in college students wishing to solve an interpersonal conflict with friends or family members (Oettingen et al., 2001, Studies 1 and 3), to get to know an attractive stranger (Oettingen, 2000, Study 1), or to achieve self-improvement (e.g., being more assertive; Oettingen et al., 2005, Study 1).

These findings (reviews by Oettingen \& $\mathrm{Ha}$ genah, 2005; Oettingen \& Thorpe, 2006) imply that perceiving the envisioned future as desirable (positive attitude or high incentive value; Ajzen, 1991) and feasible (high perceived control or efficacy expectations; Bandura, 1997) is a just prerequisite for the emergence of strong goal commitments. To create strong goal commitments, however, people need to translate these positive attitudes and high expectations into binding goals, a process that is facilitated by mentally contrasting the positive future with negative reality.

One might argue, alternatively, that mental contrasting only raises people's expectations of success and subsequently produces stronger goal commitments. As has been pointed out in the literature, increasing the desirability of an event may boost optimism with respect to the desired event (e.g., Krizan \& Windschitl, 2007). Or one may suggest that mental contrasting and indulging lead to different levels of optimism because the two strategies construe the desired future at different levels of psychological distance (for example, Armor \& Sackett (2006) observed that temporally more distant construals led to heightened optimism), and thus differentially affect people's level of expectations of success. But it seems unlikely that juxtaposing obstacles to a desired future increases the desirability of the positive future as compared to merely indulging in it, and it seems equally unlikely that mental contrasting produces a more distal construal of the positive future than indulging. Indeed, none of the studies reported above found mental contrasting to significantly increase desirability or feasibility (expectations) of the positive future, and the observed pattern of findings remained the same even when changes in desirability and feasibility (i.e., incentive value and expectation) from before to after the manipulation of the two strategies were adjusted for. These additional results suggest that goal commitment effects of mental contrasting are produced by the instigated problemsolving orientation rather than changes in levels of desirability or feasibility of the desired future.

\section{Brain activity linked to mental contrasting and indulging}

Past research on mental contrasting and indulging primarily focused on the strength of the resulting intentions (goal commitments). Contrarily, the present study is an attempt to elucidate the postulated cognitive components and processes of mental contrasting and indulging by looking at 
brain activity in the relevant regions. In other words, brain activity is assessed to test fantasy realization theory's (Oettingen, 2000; Oettingen et al., 2001) assumption that mental contrasting is a problem-solving mental strategy of intention formation that is cognitively demanding and refers back to past experiences. On the contrary, indulging is a cognitively undemanding daydreaming strategy that is oblivious to one's performance history.

Specifically, mental contrasting, as compared to resting, should be associated with greater activity in brain regions linked to working memory processes as mental contrasting effects are based on mentally placing the present negative reality in the way of the desired future. However, mental contrasting should also lead to greater activity in brain areas associated with episodic memory because it demands the elaboration of obstacles. Such elaborations should recruit memories of relevant obstacles that were experienced in the past as well as relevant memories about past successes and failures in trying to overcome them.

Mental contrasting should also be linked to heightened activity in brain regions that are related to vividly imagining events. As the mental contrasting procedure demands switching back and forth from positive images about a desired future to images of impeding obstacles, images of both the desired future and obstacles should become vivid and crystallized. Finally, mental contrasting should lead to a greater activity in brain regions that are related to holding intentions and action preparation because mental contrasting leads to the formation of strong intentions, given that relevant expectations of success are high.

The cognitive components and processes of indulging are different from those of mental contrasting. As the problem of "Shall I realize the imagined future or not?" does not arise during indulging, no purposeful processing of information is instigated (such as disrupting one's thinking about the positive future in order to address obstacles). Rather, indulging only implies the flow of positive images about the desired future. Therefore, working memory should not be engaged, and as relevant obstacles and one's way of dealing with them do not need to be recalled, episodic memory should also not be taxed. Moreover, the images about the positive future can stay vague, as they are not tied to past or present obstacles. Finally, only moderate intentions to realize the desired future are formed, even in the face of high expectations.
In sum, as compared to a relaxed cognitive activity during rest, greater activity in the brain areas implicated in working memory, episodic memory, vivid imagery, and intention formation should be observed during mental contrasting (in which people are engaged in a task-specific, purposeful mental activity), but not during indulging (which only involves free and vague daydreaming about a desired future).

\section{Regions of interest}

Given our hypotheses, we defined the following regions of interest (ROIs): (1) prefrontal, (2) frontal, (3) temporal, (4) parietal, and (5) occipital. These five regions were additionally differentiated by hemisphere, resulting in 10 ROIs (see Figure 1). The defined regions of interest are kept relatively broad, as interindividual differences in distributions of dipoles do not allow for more precise predictions with respect to complex mental processes (Wang, Kakigi, \& Hoshiyama, 2001). Working memory, holding intentions in mind, and action preparation are all associated with prefrontal and frontal brain regions (e.g., Spence \& Frith, 1999; West, 2005; Zhu, 2003). For instance, the prefrontal cortex shows enhanced activity in fMRI studies when participants have to keep a task goal in mind while performing various subgoals (e.g., Koechlin, Basso, Pietrini, Panzer, \& Grafman, 1999), willed action like choosing between more than one appropriate response was observed to be linked to the left prefrontal cortex in a PET study (Frith, Friston, Liddle, \& Frackowiak, 1991), and frontal networks were associated with task preparation during a task-cueing paradigm in MRI studies (e.g., Brass \& von Cramon, 2002). Furthermore, it is frequently observed that the formation and realization of intentions is supported by a neural network that involves structures within the frontal and parietal cortices (see Eagleman, 2004, for review). Thus, several areas of the frontal lobe are suggested to be involved in the formation of intentions, the maintenance of intentions in working memory, and the realization of intentions (Amodio \& Frith, 2006; West, 2005; Zhu, 2003).

Episodic memory retrieval processes, on the other hand, are strongly associated with activity in the temporal lobes. In an fMRI study, Bunge, Burrows, and Wagner (2004), using a non-verbal associative retrieval task, observed that the medial-temporal lobe showed greater activity during 


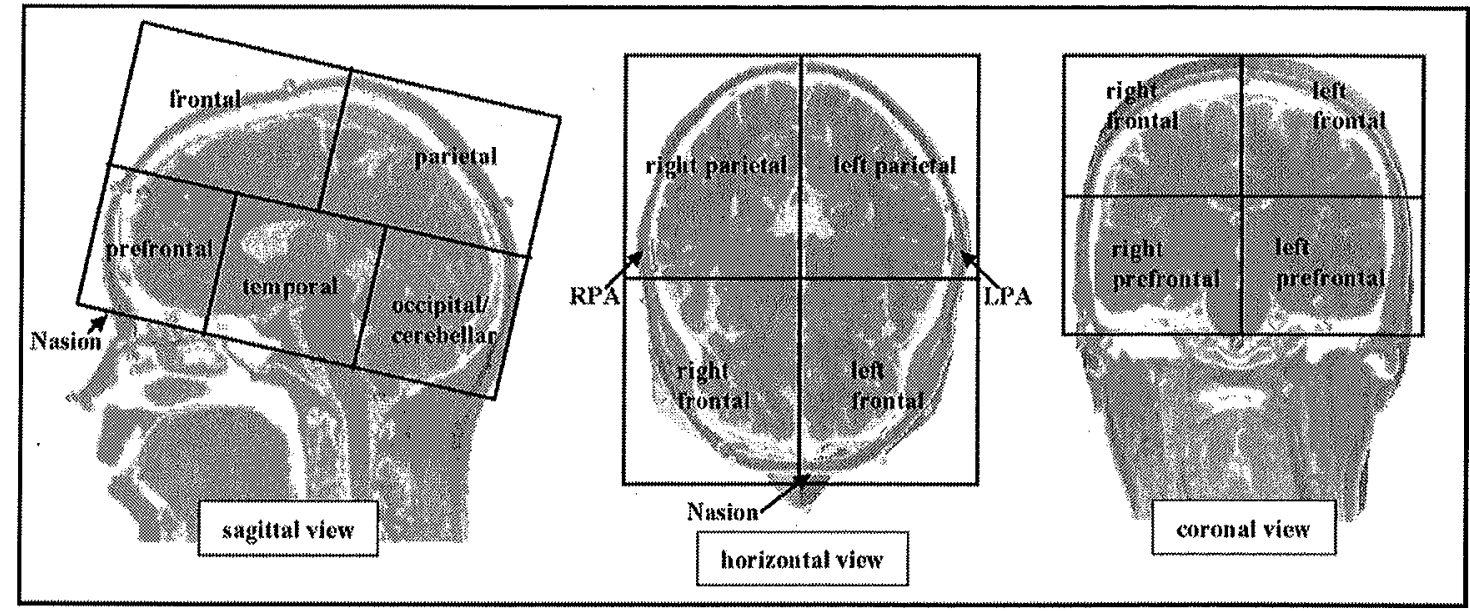

Figure 1. Schematic illustration of the 10 regions of interest (ROIs) used to cluster the MDD data.

the retrieval of strongly associated visual patterns as compared to the retrieval of weakly associated patterns. Cabeza et al. (2004) presented undergraduate students with photos they had taken by themselves in different campus locations (autobiographical condition) and similar photos taken by other persons (nonautobiographical condition). In the autobiographical condition compared to the nonautobiographical condition, the authors observed increased blood-oxygen-level dependent (BOLD) signals in the temporal lobes (especially in the hippocampus).

Parietal regions are related to the awareness and inferring of one's own intentions (e.g., Haggard, Clark, \& Kalogeras, 2002; Lau, Rogers, Haggard, \& Passingham, 2004; Oztop, Wolpert, \& Kawato, 2005). More specifically, Sirigu et al. (2004) observed that patients with parietal lesions could not indicate when they became aware of their intention to move, though they could indicate when they started to move, whereas healthy participants in the same paradigm could easily tell both the occurrence of their intention to move and its enactment. Finally, vivid visual imagery is known to be based on brain activity in the occipital regions (see Richardson, 1999).

\section{Assessment of brain activity}

In the present study, all participants were first asked to resign to a rest condition. Thereafter, they were asked to engage in both mental contrasting and indulging (order was counterbalanced). Based on the presented arguments and reported findings, we expected greater brain activity in the prefrontal and frontal cortex, as well as the temporal, parietal, and occipital regions of the brain during mental contrasting as compared to both indulging and resting. In order to assess brain activity, we analyzed continuous MEG data in a rather novel way (see the multiple dipole density method described below). MEG studies commonly determine brain activity that is triggered by the presentation of certain stimuli. Brain activity elicited by ongoing thought has been successfully analyzed by MEG investigations so far only with respect to higher mental processes in schizophrenics (Fehr, 2002; Fehr et al., 2003b).

\section{METHOD}

\section{Participants}

For the present MEG study, we selected nine participants (age: $M=23.31 ; \mathrm{SD}=2.51$ ) by using a pretest (see below). At the end of the last $M E G$ recording session, participants were debriefed on the purpose of the whole study, thanked, and paid (50 euros). Participants who participated only in the pretest were debriefed immediately thereafter, thanked, and paid (10 euros).

\section{Design}

Due to no ad hoc assumptions about laterality, the study followed a 3 within (mental strategy: rest vs. indulging vs. contrasting) $\times 10$ within (region: 
right prefrontal vs. left prefrontal vs. right frontal vs. left frontal. vs. right temporal vs. left temporal vs. right parietal vs. left parietal vs. right occipital vs. left occipital) two-factorial design. We assessed the number of dipoles per second as the dependent variable.

\section{Procedure}

\section{Pretest}

A total of 100 female participants were asked to name both their two most desired interpersonal future outcomes (participants named, for instance, getting to know an attractive fellow student) and their two most desired academic future outcomes (participants named, for instance, receiving a top grade in an upcoming exam). Subsequently, in order to measure expectations of success, participants had to judge the likelihood of achieving each of the four named desired outcomes on a scale ranging from $0 \%$ to $100 \%$.

The experimenter then handed out detailed written instructions on how to engage in the two different modes of thought with respect to their desired interpersonal outcomes (see Oettingen, 2000, Study 1; Oettingen et al., 2001). Mental contrasting instructions requested participants to list two positive aspects they associated with having attained one of the two desired interpersonal outcomes and two aspects of present reality that stand in the way of reaching this desired outcome. Thereafter, participants were asked to elaborate these aspects in the following order. First, they were told to imagine events and scenarios related to one of the positive future aspects. Specifically, participants were instructed: "Think about this aspect and depict the respective events or experiences in your thoughts as intensively as possible! Let the mental images pass by in your thoughts and do not hesitate to give your thoughts and images free reign. Take as much time and space as you need to describe the scenario. If you need more space to write, please use the back of the page." Using the same instructions, participants were then asked to imagine events and scenarios related to one of the listed aspects of negative reality. Finally, participants were asked to turn to the other listed positive future aspect, and subsequently to the other listed negative reality aspect.

Indulging instructions were equally detailed. Participants first had to list four positive aspects they associated with having attained the other of their two most desired interpersonal outcomes. Participants were then requested to mentally elaborate all four positive future aspects using the instructions cited above.

Thus, mental contrasting and indulging instructions differed only in terms of which aspects of the two interpersonal outcomes needed to be elaborated. Specifically, mental contrasting demanded alternation between the two positive aspects of the future and the two negative aspects of reality, whereas indulging demanded the elaboration of the four positive aspects of the future. Each participant had to follow the mental contrasting instructions for one, and the indulging instructions for the other of the two most desired interpersonal outcomes named. Which of the two received mental contrasting versus indulging instructions was determined by random selection, and the temporal order of the two different instructions was counterbalanced across participants. Participants performed both mental contrasting and indulging for about $10 \mathrm{~min}$ each, with a $3 \mathrm{~h}$ break between.

In order to select the final sample of nine participants for the MEG recordings, we proceeded as follows. With respect to mental contrasting, two independent raters indicated (on 9point scales) how negatively participants had elaborated the listed negative aspects of the present reality and how strongly these aspects were based on reality. Furthermore, the raters judged (on 9-point scales) how positively participants had elaborated the listed positive aspects of the desired future and how clearly these thoughts and images reached into the future. The latter rating procedure was also used with respect to judging positivity and future orientation of the indulging elaborations.

Inter-rater reliability across the 16 measures was high (all $r s>.80$ ). A composite score (i.e., the mean) using the ratings for negativity, positivity, relatedness to reality, and relatedness to the future, was computed for each participant and taken as an indicator of how well the participant did in mental contrasting; an analogous composite score was used to determine how well participants did in indulging. The two participants per group (group size ranged from 18 to 22 ) who ranked highest in both mental contrasting and indulging were invited to take part in the MEG study to be conducted the next day. Importantly, we only selected participants who also reported medium to high expectations that their most desired 
academic outcomes named at the beginning of the pretest would be attained. Mental contrasting should translate such high expectations into strong intentions (goal commitments), whereas indulging should fail to do so (Oettingen et al., 2001).

In order to prevent participants from starting to think about their two most desired academic outcomes prior to the MEG recordings, participants were informed that the MEG recordings the next day would investigate various higher cognitive functions, such as algebraic calculations and reading.

\section{$M E G$ recordings}

First, a modified Edinburgh Handedness Test was conducted with each participant to assure that all nine participants were right-handed. After the test, we recorded electromagnetic brain activity (MEG) during three time periods. During a first 5-min rest period, participants were asked to relax but to stay awake. Thereafter, participants engaged in either mental contrasting or indulging for a period of $10 \mathrm{~min}$. This was followed by a break of $1.5 \mathrm{~h}$ during which the participants left the recording room and were allowed to occupy themselves by reading daily newspapers or magazines. Accordingly, this break was controlled in terms of duration and the type of mental activity participants engaged in (i.e., reading; see Gevins et al., 1998). At the beginning of the break, participants were told that a random generator would determine which task they will have to perform next. In fact, after the break, all participants had to engage in the other strategy of intention formation. Specifically, indulging participants had to mentally contrast, and mental contrasting participants had to indulge.

Prior to the MEG recordings, the experimenter asked participants to recall the two most desired academic outcomes they had named in the pretest session. Participants then learned that during the MEG recordings they had to engage in mental contrasting and indulging with respect to their two most desired academic outcomes; this time, however, simply by performing these procedures in front of their mind's eye without writing down their thoughts and images. For each participant, the experimenter randomly chose one of the two named desired academic outcomes for mental contrasting and indulging; the temporal order of the two strategies was counterbalanced across participants.

\section{Data acquisition and analysis}

The MEG was recorded using a 148-channel whole-head neuromagnetometer (MAGNESTM 2500 WH, 4D Neuroimaging, San Diego, CA) installed in a magnetically shielded cubicle (Vacuumschmelze, Hanau, Germany). Recordings were obtained in a lying position. Throughout the recordings, to avoid eye and head movements, participants were asked to fixate on a yellow point placed at the ceiling of the cubicle. A video camera installed inside the cubicle allowed for monitoring participants' behavior and compliance. The MEG was recorded with a $678.17-\mathrm{Hz}$ sampling rate, using a bandpass filter of $0.1-200$ $\mathrm{Hz}$. For artefact control, eye movements (EOG) were recorded from four electrodes attached to the left and right outer canthus as well as above and below the right eye. The electrocardiogram (ECG) was monitored via electrodes attached to the inner sides of the two forearms. A synamps amplifier (NEUROSCAN ${ }^{\circledR}$, El Paso, TX) served for the recording of $E O G$ and $E C G$.

Interindividual differences of psychophysiological parameters require sensitive methodologies of analyzing the collected data. This is particularly true when higher cognitive functions are at issue (due to the complexity of the underlying neural networks) and relatively weak physiological signals are scrutinized (Reinvang, Greenlee, \& Herrmann, 2003). In order to meet these challenges, we resorted to a multiple dipole density procedure used on bandpass filtered continuous MEG data recorded during rest, mental contrasting, and indulging. As brain activity measured by MEG during continuing task performance is characterized by a composition of oscillations in different frequency bands, and parameters due to oscillatory brain activity show considerable individual differences, the method used in our study is based on individual task-related power spectra averaged over all $148 \mathrm{MEG}$ channels. Prominent task-related frequency bands were identified by visual inspection of the power spectra, and the original continuous data epochs were then bandpass filtered according to the identified frequency ranges. Therefore, locations for possible generators as described by dipoles of the activity were estimated by a multidipole fit strategy. Dipoles that represent a considerable part (as justified by their strength of current dipole equivalent) of the estimated models weighted the dipole density parameters (number of dipoles per second and region) according to their position in the brain. 
This procedure provides parameters that allow an estimated localization of task-related brain oscillations independent of individual differences due to oscillatory frequency ranges. More specifically, we combined a fast Fourier transformation (FFT) and a multiple dipole density (MDD) method (for details see Fehr, Achtziger, Hinrichs, \& Herrmann, 2003a).

\section{RESULTS}

\section{FFT and multiple dipole density analyses}

For each participant and mental strategy, an FFT was performed on the data. FFT results were averaged over all $148 \mathrm{MEG}$ channels. The resulting power spectra were plotted for each subject and mental strategy (see Figure 2). Power spectra were visually inspected and prominent deflections of each spectrum were noted as possible effects of generators underlying background and/or mental strategy related activity (see Figure 3 for critical frequency ranges). Original data sets were then bandpass filtered separately for all critical frequency bands $1 \mathrm{~Hz}$ around identifiable peaks in the power spectrum or for more extensive cohesive frequency ranges in which a particular mental strategy clearly showed more power than the others. Thereafter, the whole data range of each data set was visually inspected and an epoch (about $5 \mathrm{~s}$ ) in which all channels showed activity was identified. For those 5-s epochs, a stepwise multidipole analysis was performed using the software BESA2000 (www.megis.com). A spatiotemporal PCA was calculated to get a first impression of approximately how many dipoles have to be expected to explain the data in the actual model. In the next step, the epoch with the highest deflections in the eigenvalue curve was marked to fit the first dipole(s). Thereafter, additional dipoles were added one by one and fitted on partial epochs showing the highest residuals. The number of dipoles was increased until $90 \%$ of variance was explained (by a maximum of 15 dipoles, see Figure 4 for illustration). Afterwards, all dipoles were fitted simultaneously for the whole epoch to tune the position of each dipole in the model. In dipole fit procedures using the BESA software, dipoles were first set to different positions in order to find the best starting position. This prevents dipoles from being fitted to local minima. Fitting

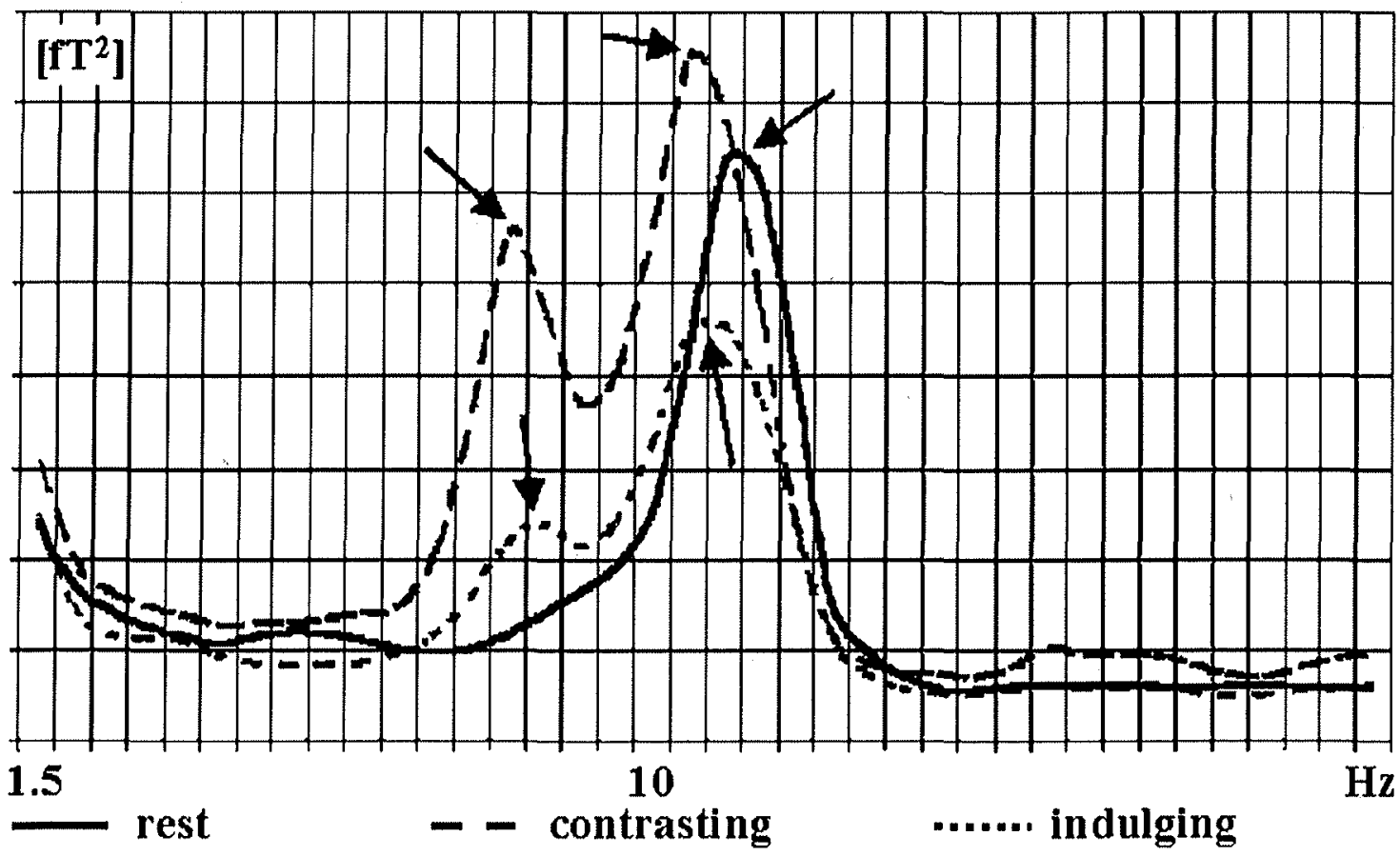

Figure 2. Example of three power spectra (rest, indulging, and mental contrasting) of one participant. The different mental strategies show several specific deflections (see arrows). 


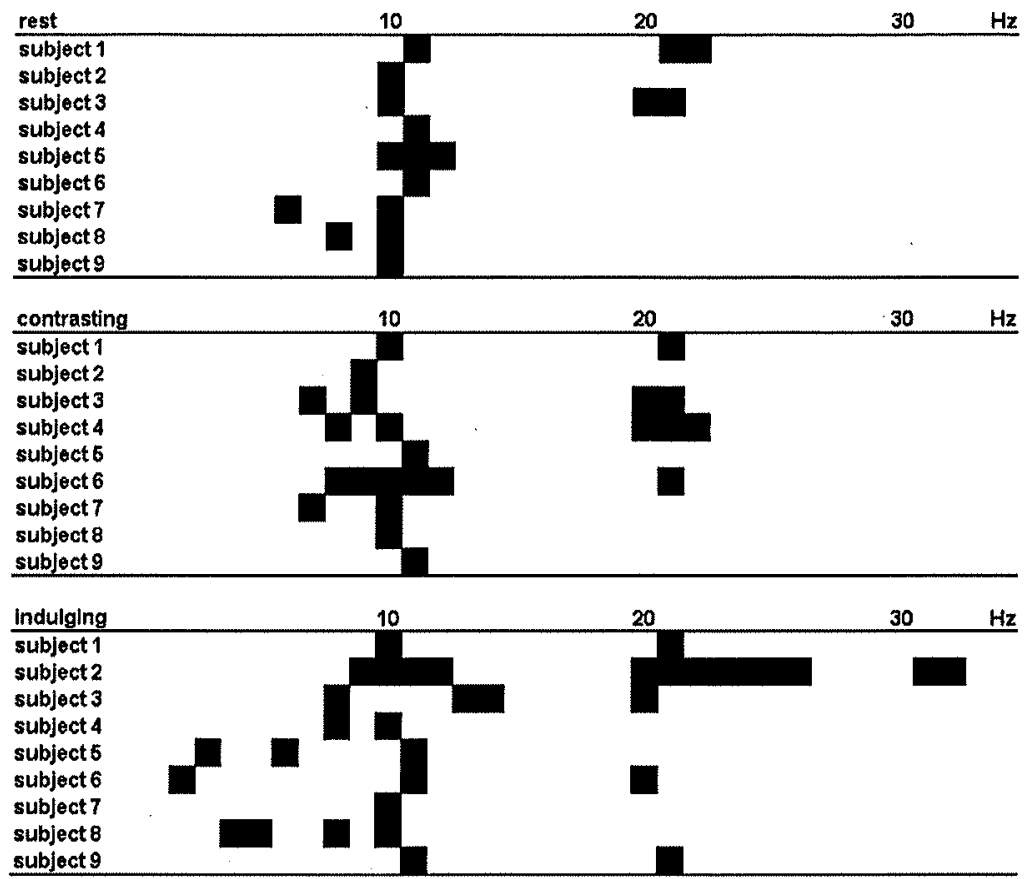

Figure 3. Frequency ranges in which prominent deflections occur were shadowed for each participant and mental strategy.

the first single dipole sometimes resulted in a dipole position located in the middle of the sphere model. Those solutions often represent a sum of source activities at different positions. This could be confirmed by the huge moment of those dipoles that could not be generated by a valid physical source in the middle of the brain. A manual arrangement of two or three dipoles in positions around the center of the sphere often provided a more appropriate starting constellation for the fitting procedure. Therefore, central dipole locations were excluded from further analyses. The multidipole model reaching the criterion explained above was then used to fit the complete data (a $60 \mathrm{~s}$ epoch low on artefacts). The resulting source wave forms served as a basis for the statistical analysis. For statistical analyses, the brain was divided into 10 ROIs (comparable to Figure 1). To define the edges of the boxes in which the dipole density values were clustered, we followed the stereotaxic proportions established by Talairach and Tournoux (1988). Since our strategy considers interindividual differences in dipole distribution, our approach is an approximation and did not refer precisely to the Talairach Atlas. As had been shown for complex mental processes, a more precise definition of ROIs could lead to less valid preassumptions due to interindividual distribution of dipoles (Wang et al., 2001).

Each data time point of the source wave forms was examined as follows: Assuming $n$ is the number of dipoles in the present model, a dipole has to reach more than the $n$th part of the sum of current equivalents at that data time point to be considered for statistical analyses. Thereafter, the regional densities of dipoles were analyzed with respect to differences between mental strategies by an ANOVA and a nonparametric permutation test. The central aspect of the described procedure consisted of bypassing the problem of high interindividual variability of activities within fixed ranges of frequency bands. Based on the assumption that participants proceed in different frequency bands but in the same ROIs due to the same mental strategy, an FFT was performed in order to determine individual task-related frequency ranges of activity for each data set. Afterwards only those frequency ranges were analyzed in terms of source analyses. Considering interindividual differences in oscillatory brain activity, consistent task-related regional variations should be related to oscillatory brain activity in individual frequency ranges.

Participants showed specific deflections in the power spectra due to the different mental strategies prominently in theta, alpha, and beta 


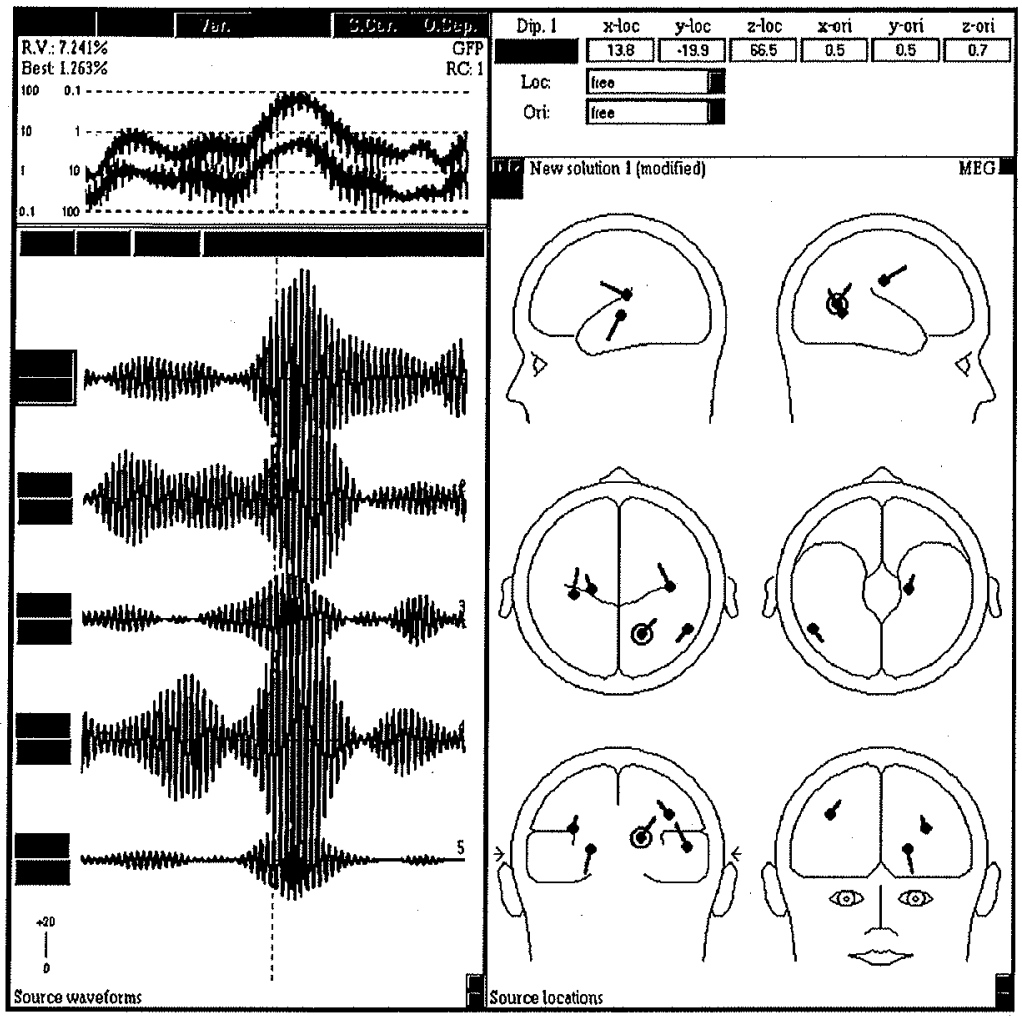

Figure 4. Source wave forms and locations of a five-dipole model based on a 6-s bandpass filtered data epoch.

frequency bands (see Figure 2). Oscillatory activity in the gamma range (above $30 \mathrm{~Hz}$ ) could not be fitted using the maximum criterion of 15 dipoles and was, therefore, excluded from further analyses. On average, $5.4( \pm 2.8)$ dipole locations were fitted per model. Participants showed 14.9 $( \pm 8.4)$ dipole locations over all models; 2.0 ( \pm $0.8)$ frequency bands were examined per mental strategy and subject.

\section{Number of dipoles per second dependent on mental strategy and brain region}

Topographical analyses by means of a 3 (Mental Strategy, within: rest vs. indulging vs. mental contrasting) $\times 10$ (Region, within: right prefrontal vs. left prefrontal vs. right frontal vs. left frontal. vs. right temporal vs. left temporal vs. right parietal vs. left parietal vs. right occipital vs. left occipital) ANOVA revealed a significant Mental Strategy $\times$ Region interaction effect, $F(18,144)=$ 2.1, $p<.01$ (uncorrected).
Physiological variables have been discussed to show deviations from Gaussian normal distribution. Due to this and our small sample size (see also Bortz \& Lienert, 1998), we further computed a nonparametric permutation test using the statistical software NPC Test(C) 2.0 (for details see www.methodologica.it, methodological Srl 2001). In order to substrate mental strategy effects depending on brain regions, we tested the following model: Mental Strategy (stratified: rest vs. indulging vs. mental contrasting) $\times$ Region (within: right prefrontal vs. left prefrontal vs. right frontal vs. left frontal. vs. right temporal vs. left temporal vs. right parietal vs. left parietal vs. right occipital vs. left occipital). This nonparametric permutation procedure confirmed the significant Mental Strategy $\times$ Region interaction ( $p<.01$, using Fisher's combining function) as reported for the parametric ANOVA procedure mentioned above. In order to elucidate this significant Mental Strategy $\times$ Region interaction effect further, differences between the three mental strategies for the different brain regions were analyzed by means of Fisher's post hoc least difference (LSD) tests. These LSD tests 
(see Table 2) revealed significantly more dipoles per second for the mental contrasting condition $(M=354, S D=649)$ than the rest condition $(M=$ $28, S D=39)$ in the left prefrontal areas, $p<.05$. Furthermore, mental contrasting generated more dipoles per second than the rest and the indulging condition in the right frontal region (mental contrasting: $M=1054, \quad S D=1084$; indulging: $M=480, S D=482$; rest: $M=430, S D=307), p<$ .01. Furthermore, in both the right and left temporal regions, mental contrasting $(M=1137$, $S D=1026)$ caused more dipoles per second than indulging ( $M=453, S D=495), p<.01$, and rest $(M=304, S D=290), p<.01$. In the right parietal region, we also observed more dipoles per second in the mental contrasting condition $(M=372$, $S D=344)$ compared to the rest $(M=35, S D=$ $47), p<.05$, and the indulging condition $(M=26$, $S D=42), p<.05$. In the right and in the left occipital regions, mental contrasting $(M=442$, $S D=509)$ significantly generated more dipoles per second than resting $(M=60, S D=74), p<.05$, and in the right occipital region more than indulging $(M=180, S D=152), p<.05$ (for details see Tables 1 and 2; for illustration see Figure 5). The indulging condition, however, did not significantly differ from the rest condition in occipital regions (i.e., the number of dipoles per second generated by indulging ranged between mental contrasting and rest; see Table 1).

\section{DISCUSSION}

Based on fantasy realization theory (Oettingen, 2000; Oettingen et al., 2001), we examined the neural substrates of the intention formation strategies of mental contrasting versus indulging by means of continuous MEG data. We tried to
TABLE 2

MDD - multiple source density; Least significant differences tests;

\begin{tabular}{|c|c|c|}
\hline Region & Left & Right \\
\hline Prefrontal & cont $>$ rest $*$ & \\
\hline Frontal & & $\begin{array}{l}\text { cont }>\text { rest } \\
\text { cont }>\text { ind }\end{array}$ \\
\hline Temporal & $\begin{array}{l}\text { cont > rest } * * \\
\text { cont > ind } * *\end{array}$ & $\begin{array}{l}\text { cont }>\text { rest } * * \\
\text { cont }>\text { ind } * *\end{array}$ \\
\hline Parietal & & $\begin{array}{l}\text { cont }>\text { rest } * \\
\text { cont }>\text { ind } *\end{array}$ \\
\hline Occipital & cont $>$ rest $*$ & $\begin{array}{l}\text { cont }>\text { rest } * * \\
\text { cont }>\text { ind } *\end{array}$ \\
\hline
\end{tabular}

Notes: rest $=$ resting; cont $=$ mental contrasting; ind $=$ indulging; * $p<.05 ; * * p<.01$

answer the following two questions: (1) Can the two mental strategies be differentiated by continuous MEG data? (2) Are the locations of greater brain activity observed in mental contrasting as compared to resting and indulging indicative of the cognitive components and processes that fantasy realization theory ascribes to mental contrasting?

First, we succeeded in differentiating the two strategies of intention formation by means of the MEG. In our study, we did not present any stimuli to evoke responses related to mental contrasting or indulging. Rather, we measured and analyzed continuous MEG activity in mental contrasting and indulging individuals who had been preselected on the basis of proficiently performing both of these mental strategies. In order to analyze the continuous MEG activity and to take into account individual differences in psychophysiological parameters, an FFT was combined with an MDD method. A new feature of the applied strategy of data analysis was to consider interindividual differences in oscillatory brain activity in relation to complex mental processing. Oscillatory neural

TABLE 1

MDD: group means of regional "dipoles per second" values and standard deviations for the 10 ROIs

\begin{tabular}{lcccc}
\hline Region & Hemisphere & Rest & Indulging & Contrasting \\
\hline Prefrontal & left & $28 \pm 39$ & $53 \pm 87$ & $354 \pm 649$ \\
Frontal & right & $109 \pm 165$ & $67 \pm 122$ & $205 \pm 328$ \\
& left & $112 \pm 150$ & $163 \pm 193$ & $253 \pm 206$ \\
Temporal & right & $430 \pm 307$ & $480 \pm 482$ & $1054 \pm 1084$ \\
& left & $226 \pm 174$ & $415 \pm 510$ & $1004 \pm 1084$ \\
Parietal & right & $383 \pm 406$ & $491 \pm 481$ & $88 \pm 98$ \\
& left & $30 \pm 60$ & $26 \pm 42$ & $1271 \pm 1068$ \\
Occipital & right & $35 \pm 47$ & $135 \pm 139$ & $372 \pm 344$ \\
& left & $45 \pm 56$ & $180 \pm 152$ & $355 \pm 350$ \\
\hline
\end{tabular}



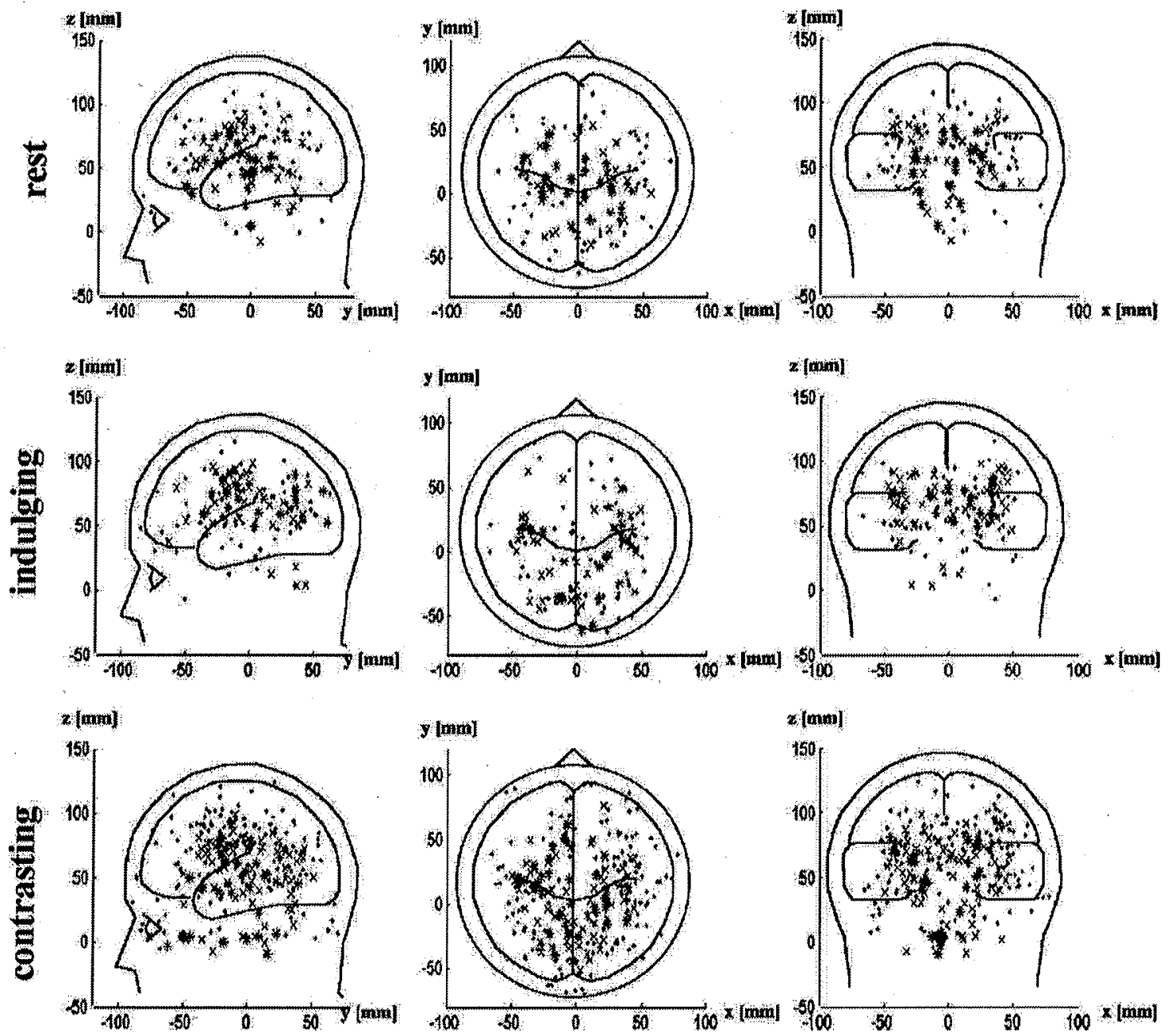

Figure 5. Plotted MDD over all participants weighted due to the source wave forms for the rest, indulging, and mental contrasting conditions.

network activity of different generators in interindividual different frequency bands have been integrated in one analysis. This procedure demonstrated an advanced and appropriate approach in the analysis of continuous multichannel MEG data of high complexity.

Second, the observed pattern of dipoles per second in the brain areas of interest suggests that mental contrasting is indeed a problem-solving strategy as specified by fantasy realization theory. Specifically, we observed more dipoles per second in the left prefrontal area during mental contrasting compared to rest, and more dipoles per second in the right frontal areas during mental contrasting compared to both indulging and rest. No difference between indulging and rest was found for either left or right prefrontal and frontal areas (the level of activation associated with indulging was always closer to resting than mental contrasting, see Table 1). We interpret this pattern of brain activity as measured by dipoles per second of prefrontal and frontal areas as an indication that mental contrasting involves working memory and the formation of intentions with respect to action preparation, in that the prefrontal and frontal cortex have been identified as the brain regions associated most closely with these mental functions and procedures (summaries by Frith et al., 1991; Spence \& Frith, 1999; West, 2005; Zhu, 2003). We are aware of research observing that differences in the brain activity in left and right prefrontal and frontal areas were dependent on approach and avoidance motivation (e.g., Harmon-Jones, 2003; Amodio, Shah, 
Sigelman, Brazy, \& Harmon-Jones, 2004). These authors, among others, showed that the activation of the left prefrontal/frontal cortex is more strongly associated with approach motivation, whereas the activation of the right prefrontal/ frontal cortex is more strongly associated with avoidance motivation. As we had no a priori assumptions regarding whether mental contrasting or indulging should lead to more approach or more avoidance motivation, we did not predict more dipoles per second for prefrontal or frontal areas depending on the hemisphere. Due to our findings, one can speculate as to whether mental contrasting leads to both approach motivation (geared towards approaching and reaching the desired future) and avoidance motivation (geared towards avoiding and circumventing the hindrances of present reality).

For temporal brain areas more dipoles per second were observed during mental contrasting as compared to both indulging and resting bilaterally. This finding suggests that mental contrasting is rooted in the retrieval of past personal events, as episodic memory is associated with temporal areas (e.g., Cabeza et al., 2004). Note that mental contrasting led to a higher activation of the temporal areas not only as compared to rest but also as compared to indulging. Apparently, indulging does not rest on episodic memory processes. This finding implies that indulging in a positive future does not entail the mental exploration of past experiences, but of the unrealized and thus not yet experienced desired positive future (Oettingen, 2000; Oettingen et al., 2001).

During mental contrasting, we also observed more dipoles per second in the right parietal regions compared to both indulging and rest. This is in line with the intention formation hypothesis of mental contrasting, as one of the functions of the parietal cortex is the awareness as well as inferring of one's own intentions (Haggard et al., 2002; Oztop et al., 2005; Sirigu et al., 2004). The assumption of fantasy realization theory that mental contrasting leads to strong intentions in the face of high expectations of success, whereas indulging leads to only moderate intentions even when perceived probabilities of success are high, is thus supported by the observed activity pattern for the right parietal region. The observation that significantly more dipoles per second were generated by mental contrasting compared to indulging and rest in right parietal regions, whereas there was only a trend for a stronger generation of dipoles per second by mental contrasting in left parietal regions, is in line with our observation that mental contrasting generally increased activity in the right hemisphere more than indulging. The greater right parietal brain activity during mental contrasting is in accordance with observations of other authors who report greater right parietal brain activity compared to left parietal regions during tasks in which intentions of others should be inferred in an fMRI study (e.g. Kobayashi, Glover, \& Temple, 2006). Furthermore, our findings are in line with research showing that the successful realization of intentions seems to be more strongly associated with activity in right parietal regions during an fMRI session than left parietal regions (Heinze et al., 2006).

Interestingly, the observation that more dipoles per second can be found bilaterally in occipital regions during mental contrasting compared to resting, as well as in the right occipital region during mental contrasting as compared to indulging, suggests that mental contrasting indeed induces strong visual images. Greater activity of occipital regions in the MEG was observed during visual imagery of letters (Salenius, Kajola, \& Thompson, 1995) and memory search for previously seen pictures (Kaufman, Schwartz, Salustri, \& Williamson, 1990). In addition, vivid imagers showed more brain activity than nonvivid imagers in posterior regions (Richardson, 1999), and paying attention to visual stimuli presented during an experiment is reflected in greater activity of the visual cortex (e.g., measured by an increase of the BOLD signal in this area; Kastner \& Ungerleider, 2000). Nevertheless, it should be mentioned that indulging at least tended to generate more dipoles per second in occipital areas than rest. This finding suggests that indulging is associated with more mental imagery than simply resting.

Still, the heightened brain activity observed in occipital areas during mental contrasting compared to indulging (as indicated by more dipoles per second) implies that mental contrasting leads to more vivid imagery and closer attention to the imagined events than indulging. Apparently, daydreaming about a positive future seems possible without having to closely attend to the experienced images. On the contrary, mental contrasting forces a person to take a more critical look, thus making one's images crisp and vivid. This consideration is supported by our finding that with respect to the right occipital region, mental 
contrasting showed greater activity than indulging. In a study by Fehr et al. (2003b), participants instructed to imagine taking a walk through the park (without being presented stimuli) also showed prominently right (rather than left) hemispheric brain activity.

In sum, the findings of the present study indicate that the intention formation strategy of mental contrasting differs from resting in that it is associated with a very active brain. Mental contrasting seems to fulfil its task of turning high expectations into strong intentions by engaging working memory, episodic memory, and vivid imagery. The intention formation strategy of indulging, on the contrary, does not differ from resting, suggesting that indulging indeed involves no more than passively experiencing the desired future in the mind's eye; thus intentions of only moderate strength are formed even in the face of high expectations of success.

So far, research on fantasy realization theory has focused on testing the cognitive, affective, and behavioral consequences of using different mental strategies of intention formation. Instead, the present findings speak to the cognitive processes on which mental contrasting and indulging are based. They support the assumption of fantasy realization theory that mental contrasting is a problem-solving procedure (Newell \& Simon, 1972) involving the objective problem space (the desired future and the reality that needs to changed to get there), while indulging considers only half of the problem (the desired future). Thus, only in mental contrasting does the positive future appear as something to be achieved and the present reality as something to be changed, leading to the consideration of high expectations of success in intention formation.

\section{Potential limitations}

One may wonder whether the break between the MEG recording of the two strategies may have produced the observed brain activity differences. During this break, participants were allowed to freely choose which newspaper or magazine they liked to read. This should not have influenced the results for the following reasons: First, we counterbalanced the order of the mental contrasting and the indulging instructions; and second, as participants engaged in reading different newspapers and magazines, no specific cognitions should have been made accessible during the break (i.e., the content of the read articles ranged from politics and science to fashion). Therefore, the observed pattern of brain activity should not be a result of systematic influences stemming from the participants' activities during the break (for similar arguments see Solhjoo, Nasrabadi, \& Golpayegani, 2005; Gevins et al., 1998).

In the present study, we chose to focus on rather broadly defined ROIs, as we put two aspects into the focus of interest: First, the employed method of analyzing MEG data emphasized the individual power distribution (oscillatory brain activity), which is an advanced methodological aspect. Second, the spatial distribution has been handled in accordance with the expected activity foci in the brain. As Wang et al. (2001) observed interindividual differences in the distribution of dipole activity across fronto-temporal brain regions using a two-dipole model even for time-locked (i.e., not continuous) data in cognitive tasks (the Wisconsin Card Sorting Test (WCST) and task switching) requiring complex executive mental processing, we also expected a typical task-related but somewhat interindividually different distribution of dipole activity in the present study. Therefore, a clustering of activities as indicated by dipoles per second in larger defined ROIs seemed to be necessary to appropriately consider interindividual differences in the organization of perceptual and executive cognitions (e.g., Fuster, 2006) assumed to be required for complex mental processes as examined in the present study.

The observed results have important social implications. Even though the present study used participants' most desired future academic outcomes to explore the neural correlates of mental contrasting versus indulging, it can be assumed that mental contrasting and indulging of desired social future outcomes would reveal the same pattern of data. After all, research on fantasy realization theory has shown that mental contrasting effectively translates high expectations of success into strong goal commitments not only for desired academic or self-improvement outcomes (e.g., getting better grades, Oettingen et al., 2001, Study 4; smoking less, Oettingen, Mayer, \& Thorpe, 2007) but also for desired interpersonal outcomes (e.g., getting to know an attractive stranger, Oettingen, 2000, Study 1; resolving an interpersonal conflict, Oettingen et al. 2001, Studies 2 and 3; relating better to stigmatized peers; Oettingen et al., 2005, Study 2). Moreover, Oettingen, Barry, Guttenberg, and Gollwitzer (2007) recently found that 
college students who were asked to use the intention formation strategy of mental contrasting to manage their daily goal pursuits reported improved self-discipline and self-esteem after a 2 week intervention. These results even held when demographics and measures of wellbeing (e.g., depression, life satisfaction, and perceived stress) were controlled. Assuming that heightened selfdiscipline and self-esteem facilitate attaining desired social outcomes, there seems to be a second, indirect route by which mental contrasting helps a person to meet her social goals.

Going beyond prior research on the effects of mental contrasting on the attainment of social goals, the present findings suggest that certain preliminaries have to be fulfilled so that mental contrasting unfolds its beneficial effects. For example, as mental contrasting taxes working memory, people should not be able to effectively perform mental contrasting whenever cognitive resources are blocked by dual task activities (e.g., being occupied by demanding cognitive tasks, coping with interpersonal stressors, extreme tiredness, or physical frailty and pain). Moreover, as mental contrasting is based on the effective retrieval of relevant obstacles experienced in the past, it should be particularly effective for people who have carefully encoded past experiences with obstacles that thus can easily and accurately be retrieved from memory.

\section{CONCLUSION}

The present study analyzed continuous MEG data by using an MDD procedure on individual bandpass filtered data sets. The MDD (as parameterized by fitted dipoles per second) was based on the individual task-related deflections in the power spectra, and subsequent multidipole analyses led to a solution that allowed good regional characterization of two different strategies of intention formation: mental contrasting and indulging. We assumed that complex cognitive processes are represented by a composition of different oscillatory generators organized in complex networks in the brain. The present study therefore explored whether multiple generator (dipole) density in different individual task-related frequency ranges is indeed consistently related to a task-specific spatial MDD distribution.

The finding that mental contrasting and indulging can be differentiated by continuous MEG data recommends this methodology for the analy- sis of further strategies of intention formation as suggested by the goal literature (e.g., if-then planning, priming, committing to assigned goals; summary by Gollwitzer \& Moskowitz, 1996; Oettingen \& Gollwitzer, 2001). In such future studies, in a first session the respective strategies should be induced, and in a second session these strategies should be performed while continuous MEG data are taken. Following this procedure, one may even start to address the quantification and qualification of the temporal dynamics of the application of these strategies. Second, our findings suggest that obtaining continuous MEG data allows for testing hypotheses about the cognitive processes on which the mental strategies of intention formation under scrutiny are based. This possibility is very fortunate as self-reports on such processes are mostly unreliable (Wilson, 2002).

\section{REFERENCES}

Ajzen, I. (1991). The theory of planned behavior. Organizational Behavior and Human Decision Processes, 50, 179-211.

Amodio, D. M., \& Frith, C. D. (2006). Meeting of minds: The medial frontal cortex and social cognition. Nature Reviews Neuroscience, 7, 268-277.

Amodio, D. M., Shah, J. Y., Sigelman, J., Brazy, P. C., \& Harmon-Jones, E. (2004). Implicit regulatory focus associated with asymmetrical frontal cortical activity. Journal of Experimental Social Psychology, 40, 225-232.

Armor, D. A., \& Sackett, A. M. (2006). Accuracy, error, and bias in predictions for real versus hypothetical events. Journal of Personality and Social Psychology, 91, 583-600.

Bandura, A. (1997). Self-efficacy: The exercise of control. New York: Freeman.

Blakemore, S. J., \& Frith, C. (2003). Self-awareness and action. Current Opinion in Neurobiology, 13, 219224

Bortz, J., \& Lienert, G. A. (1998). Kurzgefaßte Statistik für die klinische Forschung. Ein praktischer Leitfaden für die Analyse kleiner Stichproben. Heidelberg, Germany: Springer.

Brass, M., \& von Cramon, D. Y. (2002). The role of the frontal cortex in task preparation. Cerebral Cortex, $12,908-914$.

Bunge, S. A., Burrows, B., \& Wagner, A. D. (2004). Prefrontal and hippocampal contributions to visual associative recognition: Interactions between cognitive control and episodic retrieval. Brain and Cognition, 56, 141-152.

Cabeza, R., Prince, S. E., Daselaar, S. M., Greenberg, D. L., Budde, M., Dolcos, F., et al. (2004). Brain 
activity during episodic retrieval of autobiographical and laboratory events: An fMRI study using a novel photo paradigm. Journal of Cognitive Neuroscience, 16, 1583-1594.

Dweck, C. S. (2000). Self-theories: Their role in motivation, personality, and development. Philadelphia: Psychology Press.

Eagleman, D. M. (2004). Perceived luminance depends on temporal context. Nature, $428,854-856$.

Fehr, T. (2002). Lokalisation langsamer Hirnaktivität bei schizophrenen Patienten mittels magnetenzephalografischer Untersuchungen und Exploration von Zusammenhängen zwischen langsamwelliger Hirnaktivität und Symptomatik. Aachen, Germany: Shaker

Fehr, T., Achtziger, A., Hinrichs, H., \& Herrmann, M. (2003a). Interindividual differences in oscillatory brain activity in higher cognitive functions-Methodological approaches in analyzing continuous MEG data. In I. Reinvang, M. W. Greenlee, \& M. Herrmann (Eds.), The cognitive neuroscience of individual differences (pp. 101-120). Oldenburg, Germany: bis-Publishers.

Fehr, T., Kissler, J., Wienbruch, C., Moratti, S., Elbert, T., Watzl, H., et al. (2003b). Source distribution of neuromagnetic slow wave activity in schizophrenic patients-effects of activation. Schizophrenia Research, 63, 63-71.

Frith, C. D., Friston, K., Liddle, P. F., \& Frackowiak, R. S. (1991). Willed action and the prefrontal cortex in man: A study with PET. Proceedings: Biological Sciences, 244, 241-246.

Fuster, J. M. (2006). The cognit: A network model of cortical representation. International Journal of Psychophysiology, 60, 125-132.

Geyins, A., Smith, M. E., Leong, H., McEvoy, L., Whitfield, S., Du, R., et al. (1998). Monitoring working memory load during computer-based tasks with eeg pattern recognition methods. Human Factors, 40, 79-91:

Gollwitzer, P. M., \& Bargh, J. A. (Eds.).(1996). The psychology of action: Linking cognition and motivation to behaviour. New York: Guilford Press.

Gollwitzer, P. M., \& Moskowitz, G. B. (1996). Goal effects on action and cognition. In E. T. Higgins \& A. W. Kruglanski (Eds.), Social psychology: Handbook of basic principles (pp. 361-399). New York: Guilford Press.

Haggard, P., Clark, S., \& Kalogeras, J. (2002). Voluntary action and conscious awareness. Nature $\mathrm{Neu}$ roscience, $5,382-385$.

Harmon-Jones, E. (2003). Clarifying the emotive functions of asymmetrical frontal cortical activity. Psychophysiology, 40, 838-848.

Heinze, S., Sartory, G., Müller, B. W., de Greiff, A., Forsting, M., \& Jüptner, M. (2006). Neural activation during successful and unsuccessful verbal learning in schizophrenia. Schizophrenia Research, 83, 121-130.

Higgins, E. T. (1997). Beyond pleasure and pain. American Psychologist, 52, 1280-1300.

Higgins, E. T., \& Sorrentino, R. M. (Eds.). (1990). Handbook of motivation and cognition: Foundations of social behavior (Vol. 2). New York: Guilford Press.

Kastner, S., \& Ungerleider, L. G. (2000). Mechanisms of visual attention in the human cortex. Annual Review of Neuroscience, 23, 315.

Kaufman, L., Schwartz, B., Salustri, C., \& Williamson, S. J. (1990). Modulation of spontaneous brain activity during mental imagery. Journal of Cognitive Neuroscience, 2, 24-132.

Kobayashi, C., Glover, G. H., \& Temple, E. (2006). Children's and adults' neural bases of verbal and nonverbal "theory of mind". Neuropsychologia, 45, $1522-1532$

Koechlin, E., Basso, G., Pietrini, P., Panzer, S., \& Grafman, J. (1999). The role of the anterior prefrontal cortex in human cognition. Nature, 399, 148 151.

Krizan, Z., \& Windschitl, P. D. (2007). The influence of outcome desirability on optimism. Psychological Bulletin, 133, 95-121.

Lau, H. C., Rogers, R. D., Haggard, P., \& Passingham, R. E. (2004). Attention to intention. Science, 303, 1208-1210.

Methodoligica Srl. (2001). NPC(C) 2.0 - Statistical software for multivariate permutation tests. Treviso, Italy: Methodologica srl (http://www.methodologia. it).

Morsella, E., Bargh, J. A., \& Gollwitzer, P. M. (Eds.). (in press). The psychology of action (Vol. 2): Mechanisms of human action. Oxford: Oxford University Press.

Newell, A., \& Simon, H. A. (1972). Human problem solving. Englewood Cliffs, NJ: Prentice Hall.

Oettingen, G. (2000). Expectancy effects on behavior depend on self-regulatory thought. Social Cognition, $18,101-129$.

Oettingen, G., Barry, H., Guttenberg, K. B., \& Gollwitzer, P. M. (2007). Self-discipline and self-esteem: A mental contrasting with implementation intentions intervention. Submitted for publication.

Oettingen, G., \& Hagenah, M. (2005). Fantasies and the self-regulation of competence. In A. Elliot \& C. Dweck (Eds.), Handbook of competence and motivation (pp. 647-665). New York: Guilford Press.

Oettingen, G., \& Gollwitzer, P. M. (2001). Goal setting and goal striving. In A. Tesser \& N. Schwarz (Eds.), Intraindividual processes. Volume 1 of the Blackwell Handbook in Social Psychology (pp. 329-347). Oxford: Blackwell.

Oettingen, G., Hönig, G., \& Gollwitzer, P. M. (2000). Effective self-regulation of goal attainment. International Journal of Educational Research, 33, 705732 .

Oettingen, G., Mayer, D., \& Thorpe; J. S. (2007). Mental contrasting and smoking secession. Unpublished manuscript, University of Hamburg, Hamburg, Germany.

Oettingen, G., Mayer, D., Thorpe, J. S., Janetzke, H., \& Lorenz, S. (2005). Turning fantasies about positive and negative futures into self-improvement goals. Motivation and Emotion, 29, 236-266.

Oettingen, G., Pak, H., \& Schnetter, K. (2001). Selfregulation of goal setting: Turning free fantasies 
about the future into binding goals. Journal of Personality and Social Psychology, 80, 736-753.

Oettingen, G., \& Thorpe, J. S. (2006). Fantasy realization and the bridging of time. In L. A. Sanna \& E. C. Chang (Eds.), Judgments over time: The interplay of thoughts, feelings, and behaviors. Oxford: Oxford University Press.

Oztop, E., Wolpert, D., \& Kawato, M. (2005). Mental state inference using visual control parameters Cognitive Brain Research, 22, 129-151.

Reinvang, I., Greenlee, M. W., \& Herrmann, M. (2003). The cognitive neuroscience of individual differences. Oldenburg, Germany: bis-Publishers.

Richardson, J. T. E. (1999). Imagery. Hove, UK: Psychology Press.

Salenius, S., Kajola, M., \& Thompson, W. L. (1995). Reactivity of magnetic parieto-occipital alpha rhythm during visual imagery. Electroencephalography and Clinical Neurophysiology, 95, 453-462.

Sirigu, A., Daprati, E., Ciancia, S., Giraux, P., Nighoghossian, N., Posada, A., et al. (2004). Altered awareness of voluntary action after damage to the parietal cortex. Nature Neuroscience, 7, 80-84.

Solhjoo, S., Motie Nasrabadi, A., \& Golpayegani, M. R. H. (2005). EEG-based mental task classification in hypnotized and normal subjects. Engineering in Medicine and Biology Society, 2041-2043.

Sorrentino, R. M., \& Higgins, E. T. (Eds.). (1986). Handbook of motivation and cognition: Foundations of social behavior (Vol. 1). New York: Guilford Press.

Spence, S. A., \& Frith, C. D. (1999). Towards a functional anatomy of volition. Journal of Consciousness Studies, 6, 11-29.

Talairach, J., \& Tournoux, P. (1988). Co-planar stereotaxic atlas of the human brain. New York: Thieme.

Wang, L., Kakigi, R., \& Hoshiyama, M. (2001). Neural activities during Wisconsin Card Sorting Test MEG observation. Cognitive Brain Research, 12, 19-31.

West, R. (2005). The neural basis of age-related declines in prospective memory. In R. West (Ed.), Cognitive neuroscience of aging: Linking cognitive and cerebral aging (pp. 246-264). New York: Oxford University Press.

Wilson, T. D. (2002). Strangers to ourselves. Cambridge, MA: Harvard University Press.

Zhu, J. (2003). Locating volition. Consciousness and Cognition, 13, 302-322. 